\title{
Ampliación de la presencia del caracol africano gigante Acathina fulica (Bowdich 1822) (Mollusca: Gastropoda-Achatinidae) en la zona norte del departamento de Sucre, Colombia
}

\section{Extension of the African giant snail's Acathina fulica (Bowdich 1822) (Mollusca: Gastropoda-Achatinidae) presence in the north area of the Sucre department, Colombia}

De La Ossa-Lacayo Alejandro ${ }^{1 *}$ M.Sc, Castro-Carmona José2 Zootec, Monroy-Pineda María ${ }^{3}$ M.Sc.

${ }^{1}$ Selvagua S.A.S. Universidad de Sucre, Grupo de Investigación en Biodiversidad Tropical, Colombia. ${ }^{2}$ Corporación Autónoma Regional de Sucre CARSUCRE, Colombia.

${ }^{3}$ Universidad de Sucre, Departamento de Ingeniería Civil, Colombia.

Keywords:

CARSUCRE;

African giant snail;

presence;

Sucre;

Colombia.

\section{Abstract}

The present work determines the presence of the African giant snail (Acathina fulica), in the north area of the Sucre department, in jurisdiction of the Corporación Autónoma Regional de Sucre - CARSUCRE. Density and ranges of sizes were established for each sites sampled: Sincelejo, Corozal y Tolú; the control protocols indicated by the respective environmental authority were followed and the potential impacts that this invasive species could cause were discussed.
Palabras Clave:

CARSUCRE; caracol gigante africano; presencia;

Sucre;

Colombia.

\section{Resumen}

El presente trabajo establece la presencia del caracol gigante africano (Acathina fulica), en la zona norte del departamento de Sucre, en jurisdicción de la Corporación Autónoma Regional de Sucre - CARSUCRE. Se determinó densidad y rangos de tallas para cada localidad muestreada: Sincelejo, Corozal y Tolú, igualmente se siguieron los protocolos de control indicados por la autoridad ambiental respectiva y se discuten los posibles impactos que esta especie invasora podría ocasionar. 


\section{Introducción}

La familia Achatinidae posee los caracoles terrestres de mayor tamaño conocidos; con 13 géneros, esta familia endémica de África cuenta entre sus miembros a la especie Acathina fulica (Figura 1) que posee gran relevancia mundial por su carácter invasor (MARTÍNEZ y MARTÍNEZ, 1997; PRASAD et al., 2004; LIBORIA et al., 2009). A fulica es originario de Kenia y Tanzania (PRASAD et al., 2004), igualmente se le reporta naturalmente desde Etiopía hasta Mozambique (DORST, 1973). Según MARTíNEZESCARBASSIERE et al. (2008), su dispersión por regiones tropicales y subtropicales del mundo es muy amplia, actualmente se le reporta en todos los continentes en climas tropicales y subtropicales (RAUT y BARKER, 2002).

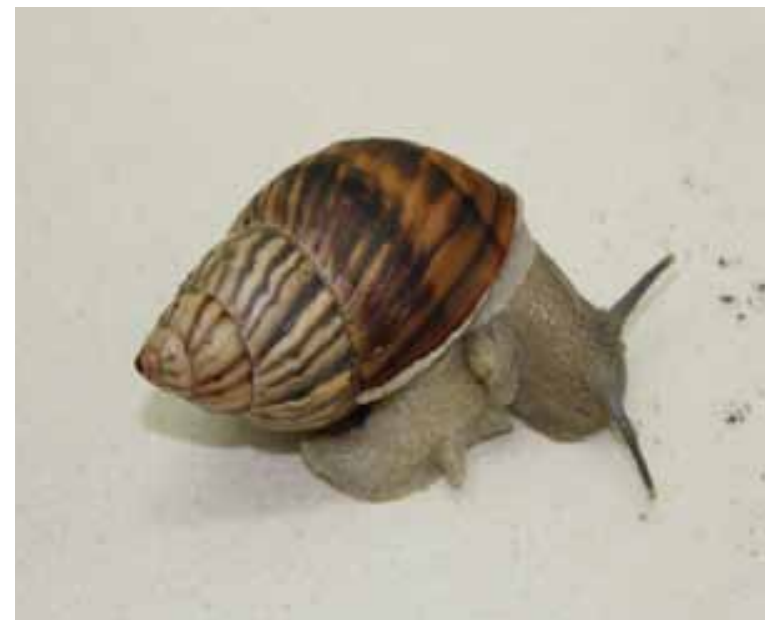

Figura 1. Muestra de ejemplares de $A$. fulica colectado durante este estudio.

Autor: Alejandro De La Ossa-Lacayo.

De acuerdo con DE LA OSSA-LACAYO et al. (2012) En Colombia se registra la presencia de A. fúlica desde el años 2010 en los siguientes departamentos: Amazonas, Antioquia, Arauca, Boyacá, Caquetá, Casanare, Guainía, Huila, Meta, Nariño, Putumayo, Santander, Tolima, Valle del Cauca, Vaupés, Guaviare, Meta, Putumayo, Tolima, Santander y Sucre. Hallazgos posteriores lo ubican también en Atlántico, Bolívar, Caldas, Córdoba, Casanare, Cundinamarca, Quindío y Vichada (ICA, 2013); De manera puntual para el departamento de Sucre se tiene registro de su presencia en Sincelejo (DE LA OSSA-LACAYO et al., 2012) y en Sampués (DE LA OSSA-LACAYO y DE LA OSSA, 2014).
Puede llegar a $30 \mathrm{~cm}$ de longitud total de la concha (VINCl et al., 1998). Entre los cinco y seis meses de edad alcanza la madurez sexual; ovíparo, hermafrodita de fertilización cruzada, con capacidad de almacenamiento de esperma para posterior fertilización (MEAD, 1949). La puesta sucede 20 días después de la cópula (LANGE, 1950); la postura está entre 100 y 200 huevos durante el primer año, valor que se sobrepasa los 500 huevos después de dos años de vida, con un rango máximo de 900 a 1200 huevos/ año (JARRETT, 1931; VAN WEEL, 1949). La eclosión sucede en un tiempo medio de 11 días (RAUT y BARKER, 2002). Se determina que posee relación directa entre la talla y el tamaño de la postura (TOMIYAMA y MIYASHITA, 1992). En medio natural alcanza un promedio de vida de 4,5 años (PLUMMER, 1975), alcanzando en cautiverio hasta 10 años (VAN LEEUWEN, 1932).

En cuanto a su dieta en medio natural, se le reconoce como herbívoro de amplio espectro, se alimenta de por lo menos 500 especies de plantas, que incluyen cultivariedades que son alimento de humanos; igualmente se reporta que come algas y líquenes; también se sabe que consume huesos, carroña, rocas calizas y paredes, que le sirven de fuente minerales (PRASAD et al., 2004; AQUINO, 2010; DE LA OSSA-LACAYO et al., 2012).

Se le reconoce como huésped intermediario de Angiostrongylus cantonensis y Angiostrongylus costaricensis, que ocasionan en humanos por infección accidental, meningoencefalitis eosinofílica y angiostrongiliasis abdominal, respectivamente (THIENGO y FERNÁNDEZ, 2005; NEUHAUSS et al. 2007, SABINA-MOLINA et al. 2009, MALDONADO et al. 2010).

En el presente estudio se registró la presencia de A. fúlica en las ciudades de Sincelejo, Corozal y Tolú, pertenecientes a la jurisdicción de la Corporación Autónoma de Sucre - CARSUCRE; se estableció el número de individuos, se tomaron medidas morfométricas y se calculó la densidad. Igualmente, se desarrollaron labores de control y se ofreció, por parte de la autoridad ambiental información sobre aspectos fundamentales a tener en cuenta para el adecuado manejo de las poblaciones detectadas en este trabajo. 


\section{Materiales y métodos}

Área de estudio: Se trabajó en tres zonas, previa información de las comunidades afectadas, en la ciudades de Sincelejo $\left(9^{\circ} 18^{\prime} \mathrm{N}\right.$ y $\left.75^{\circ} 24^{\prime} \mathrm{O}\right)$, Corozal $\left(9^{\circ} 19^{\prime} \mathrm{N}\right.$ y $75^{\circ} 17^{\prime}$ O) y Sampués $\left(9^{\circ} 31^{\prime} \mathrm{N}\right.$ y $\left.75^{\circ} 35^{\prime} \mathrm{O}\right)$.

Colecta y morfometría: Los animales fueron colectados manualmente y se dispuso de ellos siguiendo los protocolos establecidos para tal fin (MAVDT, 2011) (Figura 2). La búsqueda se llevó a cabo específicamente en las áreas en donde la comunidad reportaba observaciones, para lo cual se revisaron durante el proceso: coberturas vegetales, jardines, grietas, escombros y depósitos de desechos sólidos; la captura incluyó individuos de todas las tallas (AVENDAÑO y LINARES, 2015) (Figura 3). Los especímenes fueron medidos en la longitud de la concha (LC) mediante un calibrador pie de rey $(0,01 \mathrm{~mm})$ (AVENDAÑO y LINARES, 2015).

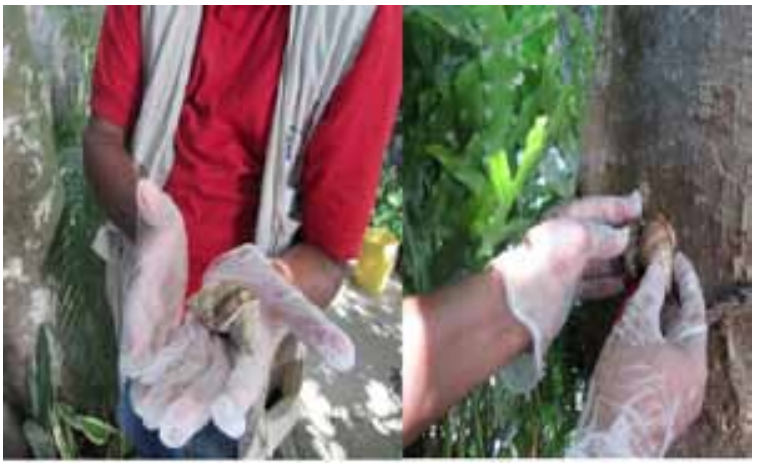

Figura 2. Manipulación en campo de ejemplares de $A$. fulica, llevada a cabo en el presente estudio. Autor: José Castro-Carmona (CARSUCRE).

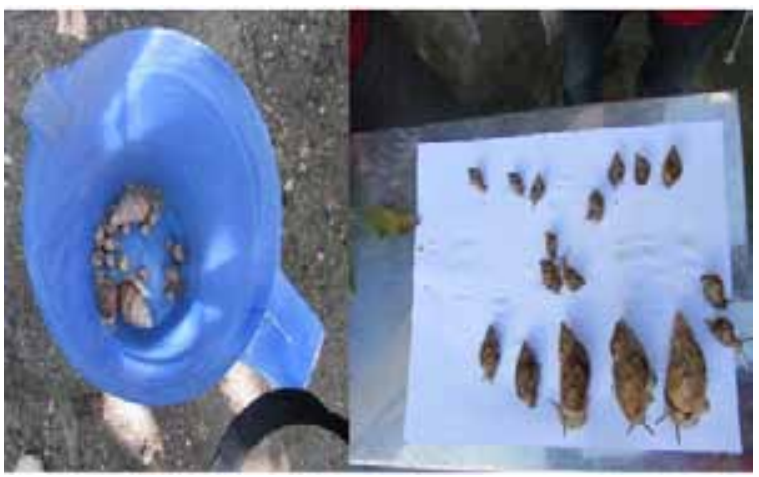

Figura 3. Muestra de las diferentes tallas de ejemplares colectados de $A$. fulica.

Fuente: Autor: José Castro-Carmona (CARSUCRE).
Calculo de densidad: se estableció de acuerdo al número total de individuos capturados por área efectiva de muestreo (DE LA OSSA-LACAYO y DE LA OSSA, 2014).

Análisis de la información: Los datos fueron organizados en tablas y previa verificación de los postulados paramétricos se aplicó mediante programa R: Anova y prueba de Tukey, con el fin de establecer diferencias entre las tres áreas muestreadas respecto de la talla de los animales. Estadísticamente el modelo matemático usado fue $\mathrm{Y}_{\mathrm{i} J}=\mathrm{U}+\mathrm{T}_{\mathrm{i}}+\mathrm{C}_{\mathrm{i}}$. Donde: $\mathrm{U}=$ media general, $\mathrm{T}_{\mathrm{i}}=$ efecto de la localidad, $\mathrm{C}_{\mathrm{iJ}}=$ =error aleatorio asociado a la iésima localidad.

\section{Resultados y discusión}

La información sobre número de animales por localidad, longitud media de la concha, peso medio, densidad se presenta en la Tabla 1.

Tabla 1. Información poblacional para cada una de las localidades muestreadas. $\mathrm{n}=$ número de individuos, LC=Longitud de la concha, DS=desviación estándar.

\begin{tabular}{ccccccccc}
\hline & Localidad & $\mathbf{n}$ & $\begin{array}{c}\text { LC X } \\
(\mathbf{c m})\end{array}$ & $\begin{array}{c}\text { Máx. } \\
(\mathbf{c m})\end{array}$ & $\begin{array}{c}\text { Mín. } \\
(\mathbf{c m})\end{array}$ & DS & $\begin{array}{c}\text { Área } \\
\left(\mathbf{m}^{2}\right)\end{array}$ & $\begin{array}{c}\mathbf{D} \\
\left(\mathbf{I n d} / \mathbf{m}^{2}\right)\end{array}$ \\
\hline Sincelejo & 78 & 27,5 & 43,2 & 18,9 & 6,29 & 3.000 & 0,026 \\
& & & & & & & \\
Corozal & 84 & 34,0 & 45,2 & 21,6 & 5,91 & 846 & 0,099 \\
Tolú & 77 & 26,4 & 39,5 & 12,4 & 6,25 & 375 & 0,205 \\
\hline
\end{tabular}

Las densidades estimadas en este estudio son inferiores al valor de $10 \mathrm{ind} / \mathrm{m}^{2}$ que se establece para áreas altamente afectadas (TILLIER, 1982); pero es superior a lo hallado por DE LA OSSALACAYO y DE LAS OSSA (2014) para Sampués y Sincelejo, en donde es registro de densidad está entre 0,0031 y $0,044 \mathrm{ind} / \mathrm{m}^{2}$, respectivamente. La diferencia comparada de las densidades para Sincelejo muestra un incremento del $550 \%$ en un lapso de 3 años.

En cuanto a la longitud de la concha, los valores obtenidos para las poblaciones de tres localidades presentan diferencias significativas: $\mathrm{df}=2$, cuadrado de la media=1389,73. $F=38,82, P<0,001$. Al aplicar prueba de Tukey se diferencia la población de Corozal, donde efectivamente la media de la 
talla es superior a la de las otras dos localidades analizadas (Tabla 2). Comparativamente, todos los individuos colectados y con referencia a las tres localidades, están por debajo de la talla máxima establecida para la especie, que oscila entre 10 y $30 \mathrm{~cm}$ (GODAN, 1983; RAUTH y GHARA, 1990; VINCl et al., 1998).

Tabla 2. Prueba de Tukey para comparar la longitud total de las tres localidades muestreadas.

\begin{tabular}{ccccc}
\hline Localidades & $\begin{array}{c}\text { Diferencia } \\
\text { entre medias }\end{array}$ & \multicolumn{4}{c}{$\begin{array}{c}\text { 95\% Limites de } \\
\text { confianza }\end{array}$} & \\
\hline Corozal- & 6.5539 & 4.2754 & 8.8325 & $* * *$ \\
$\begin{array}{c}\text { Sincelejo } \\
\text { Corozal-Tolú }\end{array}$ & 7.6242 & 5.3380 & 9.9104 & $* * *$ \\
Sincelejo-Tolú & 1.0703 & -1.2576 & 3.3982 & \\
\hline
\end{tabular}

*** Diferencias significativa $p=0,05$.

\section{Conclusiones}

Si bien es cierto que las densidades estimadas aún no llegan a valores considerados científicamente como altamente nocivos, si es preocupante el incremento detectado para Sincelejo entre 2014 y 2017 , que es la localidad para la cual se tienen datos temporales de referencia.

La presencia de la especie en Tolú es un hallazgo que debe ser mirado con las precauciones debidas, ya que entre Sincelejo y Tolú se tiene una importante porción de los Montes de María, zona caracterizada por poseer los últimos remanentes de bosque seco tropical que persisten en el Caribe colombiano. En tal sentido, no se tiene conocimiento de los posibles efectos negativos que esta especie exótica e invasora podría ocasionarle a la flora y fauna nativa allí existente.

\section{Recomendaciones}

Se deben seguir haciendo esfuerzos para la detección y control de las poblaciones de este molusco, para lo cual es inminente la participación de las autoridades municipales y departamentales. Igualmente, se deben propiciar y apoyar investigaciones que traten, entre otros temas, sobre zoonosis y ecología poblacional.

Es importante mantener campañas de información y capacitación a las comunidades de los municipios en los cuales se detecta la presencia del caracol gigante africano, con el fin de contar con un sistema de alerta en el cual los ciudadanos sean parte activa del control adecuado y trabajen de la mano de las autoridades competentes.

Se hace imperativo iniciar un proceso de detección de poblaciones de $A$. fulica en los demás municipios del Departamento, con especial énfasis en sistemas naturales sensibles, tales como los Montes de María, la Mojama y el San Jorge, sus características ambientales, muy particulares, por cierto, podrían estar sujetas a altos impactos negativos ante la invasión de esta especie.

En todos los casos se deben seguir las recomendaciones establecidas por la Resolución 654 de 2011, emanada del Ministerio de Ambiente, Vivienda y Desarrollo Territorial; lo mismo que los protocolos establecidos a nivel regional o nacional para el control de este especie.

Sería valioso que como medida de información se tengan en cuenta las campañas establecidas por diferentes autoridades, entre los cuales cabe destacar: Vídeo CORANTIOQUIA: https://www. youtube.com/watch?v=I-7Afxm_B4Q. Plan de Acción para el Manejo, Control y Prevención del caracol gigante africano en el municipio de La Dorada: http://www.corpocaldas.gov. co/publicaciones/1446/2014/La\%20Dorada/ CONTRATO\%20192-2013.pdf. Caracol gigante africano Achatina fulica. http://www.cordoba.gov. $\mathrm{co/v1/docs/salud} \mathrm{caracol} \mathrm{gigante} \mathrm{africano}$ achatina.pdf. Antecedentes de la invasión del caracol gigante africano (Achatina fulica) en Colombia. Edgar L. Linares (2013). http:// www.cardique.gov.co/public/userFiles/file/ AntecedentesinvasionAchatinafulicaColombia. pdf. Informe especial: Caracol Gigante Africano: https://www.ica.gov.co/Periodico-Virtual/Prensa/ Informe-especial-Caracol-Gigante-Africano.aspx.

Agradecimientos: A los directivas y funcionarios de CARSUCRE por el apoyo brindado y su interés en este sensible tema. A los ciudadanos preocupados que con su participación permitieron la ubicación de zonas invadidas por esta especie de caracol. A las autoridades y funcionarios municipales que se vincularon a las labores de detección y control llevadas a cabo durante este trabajo. A la Facultad de Ciencias Agropecuarias de la Universidad de Sucre por el acompañamiento científico. 


\section{Referencias}

AQUINO, M. 2010. Achatina fulica no Brasil. REDVET, Revista Electrónica de Veterinaria 11 (9):1-7.

AVENDAÑO, J.M.; LINARES, E.L. 2015. Morfometría del caracol gigante africano Achatina fulica (Gastropoda: Achatinidae) en Colombia. Cuadernos de Investigación UNED 7 (2):287-293.

DE LA OSSA-LACAYO, A.; DE LA OSSA, V.J.; LASSO, C.A. 2012. Registro del caracol africano gigante Achatina fulica (Bowdich 1822) (Mollusca: Gastropoda-Achatinidae) en Sincelejo, costa Caribe de Colombia. Biota Colombiana 13 (2):247-252.

DE LA OSSA-LACAYO, A.; DE LA OSSA, V.J. 2014. Caracol africano gigante Achatina fulica Bowdich 1822 (Mollusca: Gastropoda-Achatinidae) en zona urbana de Sincelejo y Sampués, Sucre, Colombia. Rev. Colombiana cienc. Anim. 6 (2):310-319.

DORST, J. 1973. Antes que a Natureza Morra. São Paulo: Edgard Blücher.

GODAN, D. 1983. Pests slugs and snails. Biology and control. Berlin, Springer-Verlag, Berlin.

ICA - Instituto Colombiano Agropecuario. 2013.Informe especial: Caracol Gigante Africano. Disponible en:https://www.ica.gov.co/Periodico-Virtual/Prensa/Informe-especial-Caracol-Gigante-Africano.aspx. Conusltado: 12-06-2017.

JARRETT, V.H.C. 1931. The spread of the snail Achatina fulica to South China. Hong Kong Naturalist 2:262-264.

LANGE. W.H. 1950. Life history and feeding habits of the giant African snail on Saipan. Pacific Science 4:323-325.

LIBORIA, M.; MORALES, G.; SIERRA, C.; SILVA, I.; PINO, L.A. 2009. El caracol gigante africano Achatina fulica. Iniahoy 6:224-231.

MALDONADO, J.A.; SIMÕES, R.O.; OLIVEIRA, A.P.; MOTTA, E.M.; FERNANDEZ, M.A.; PEREIRA, Z.M.; MONTEIRO, S.S.; LOPES-TORRES, E.J.; CARVALHO-THIENGO, S. 2010. First report of Angiostrongylus cantonensis (Nematoda: Metastrongylidae) in Achatina fúlica (Mollusca: Gastropoda) from Southeast and South Brazil. Memorias del Instituto Oswaldo Cruz 105 (7):938-941.

MARTÍNEZ, R.; MARTÍNEZ, E. 1997. Notes about Achatina (Lissachatina) fulica (Bowdich, 1822), dangerous african snail (Pulmonada-Achatinidae) introduced in Venezuela. Acta Biol. De Vla. 17(1):37-40.

MAVDT- Ministerio del Ambiente y Desarrollo Territorial. 2011. Resolución 654 del 7 de abril de 2011. Colombia. Disponible en: http://ambientebogota.gov.co/c/document library/get file?uuid=ef3334f2-55f143c7-ba71-2107abaa91e2\&groupld=586236. Consultada: 10-01-2017.

MEAD, A.R. 1949. The giant snails. Atlantic Monthly 184 (2):38-42.

NEUHAUSS, E.; FITARELLI, M.; ROMANZINI, J.; GRAEFF-TEIXEIRA, C. 2007. Low susceptibility of Achatina fulica from Brazil to infection with Angiostrongylus costaricensis and $A$. cantonensis. Memórias do Instituto Oswaldo Cruz 102 (1):49-52. 
PLUMMER, J.M. 1975. Observations on the reproduction, growth and longevity of a laboratory colony of Archachatina (Calachatina) marginata (Swainson) subspecies ovum. Proceedings of the Malacological Society of London 41:395-413.

PRASAD, G.S.; SINGH, D.R.; SENANI, V.; MEDHI, R.P. 2004. Ecofriendly way to keep away pestiferous Giant African Snail, Achatina fulica Bowdich from nursery. Global Invasive Species Database. Current Science 87:1657-1659.

RAUT, S.K.; BARKER, G. 2002. Achatina fulica Bowdich and others Achatinidae pest in tropical agriculture. Págs. 55-114. En: Barker, G. (Ed.). Mollusks as croup pest. CAB Publishing. New Zealand.

RAUTH, S.K.; GHARA, T.K. 1990. Impact of individual's size on density of the snail pest Achatina fulica Bowdich (Gastropoda: Achatinidae). Bollettino-Malacologico 25 (9-12):301-306.

SABINA-MOLINA, D.; ESPINOSA-BRITO, A.; NIETO-CABRERA, R.; CHÁVEZ-TROYA, O.; ROMEROCABRERA, S.J.; DÍAZ-TORRALBAS, A. 2009. Brote epidémico de meningoencefalitis eosinofílica en una comunidad rural. Revista Cubana de Medicina Tropical 61 (1):75-81.

THIENGO, S.C.; FERNÁNDEZ, M.A. 2005. Achatina fulica in Brasil: the current situation. Tentacle 2005:13:7.

TILLIER, S. 1982. Production et cycle réproducteur de l'escargot Achatina fulica Bowdich, 1822 en Nouvelle Calédonie (Pulmonata: Stylommatophora: Achatinidae). Haliotis 12:111-122.

TOMIYAMA, K.; MIYASHITA, K. 1992.Variation of egg clutches in the Giant African snail, Achatina fulica (Ferussac) (Stylomatophpra; Achatinidae). Venus 51 (4):293-301.

VAN LEEUWEN, D. 1932. Notes and comments, conchology, Achatina fulica. Hong Kong Naturalist 3:71-79.

VAN WEEL, P.B. 1949. Some notes on the African giant snail, Achatina fulica Fer. I. On its spread in the Asiatic tropics. II. On its economic significance. III. On its biological balance and means of destruction. Chronica Naturae 104:241-243, 278-280, 335-336.

VINCI, G.K.; UNNITHAN, V.K.; SUGUNAN, V.V. 1998. Farming of the Giant African Snail, Achatina fulica. India. Central Inland Capture Fisheries Research Institute 56:1-2. 